\title{
Permanencia y deserción estudiantil: razones encontradas en la licenciatura en matemáticas de la Universidad del Tolima (Colombia)
}

\author{
Permanence and Student Dropout: Reasons Found in the Bachelor Degree in Mathematics \\ in University of Tolima
}

\author{
CASTRO-CARVAJAL, Dicleny ${ }^{1}$ \\ RODRÍGUEZ-MÁRQUEZ, Miguel ${ }^{2}$ \\ SALGADO-HORTA, Dagoberto ${ }^{3}$
}

\begin{abstract}
Resumen
Es política de las universidades públicas en Colombia, aumentar la cobertura. En ese sentido el proyecto educativo del programa de Licenciatura en Matemáticas, de la Universidad del Tolima la viene cumpliendo. Sin embargo, los esfuerzos que realizan los programas de permanencia estudiantil y bienestar universitario, no han sido suficientes; así lo evidenció este estudio con estudiantes desertores. Las categorías de Tinto (1989) coinciden con las razones de abandono, aunque las variables individuales y las socioeconómicas, son las que tuvieron mayor incidencia.

Palabras clave: deserción, educación superior, investigación cualitativa, permanencia
\end{abstract}

\begin{abstract}
The policy of the colombian public universities is to increase coverage. Correspondingly, the educative project leading by the bachelor's degree in mathematics in the University of Tolima has been accomplished it. However, our research focused in dropout students statistics found that the efforts of the student support and student permanency offices are not enough. We discovered that the students' dropout reasons match with the Tinto categories (1989), highlighting that individual and socioeconomic motives are the most common.
\end{abstract}

key words: dropouts, higher education, qualitative research, continuity

\section{Introducción}

Este estudio se abordará desde dos miradas: una, la caracterización de la deserción como problema que afecta a las Instituciones de Educación Superior, con los respectivos factores que estarían asociados y que a su vez son causas de la deserción, según experiencias de cada estudiante. La otra perspectiva es, desde las condiciones de calidad que el gobierno nacional ha creado a través de las políticas educativas de retención estudiantil que obligan a las instituciones educativas a adoptar mecanismos académicos y administrativos; asimismo utiliza herramientas estratégicas como el SPADIES (Sistema para la Prevención de la Deserción en las Instituciones de

\footnotetext{
${ }^{1}$ Profesora. Facultad de Ciencias de la Educación. Universidad del Tolima. dcastroc@ut.edu.co

${ }^{2}$ Profesor. Facultad de Ciencias Económicas y Administrativas. Universidad del Tolima. marodriguezm@ut.edu.co

${ }^{3}$ Profesor. Facultad de Ciencias de la Educación. Universidad del Tolima.dsalgadoh@ut.edu.co
} 
Educación Superior) para hacer seguimiento a la deserción y revisar las condiciones académicas, socioeconómicas, institucionales e individuales que afectan la permanencia en la educación superior.

Los campus universitarios en las últimas décadas están siendo habitados por una población estudiantil cada vez más diversa en sus orígenes sociales, étnicos, culturales y económicos, y en sus características personales (Gartner y Gallego, 2013,p.1108). Estos contextos educativos y de bienestar nacional e internacional, han sufrido transformaciones, que sugieren ser revisadas y actualizadas a partir de las orientaciones disponibles(MEN,2016).

Con el propósito de contextualizar las iniciativas gubernamentales para mejorar el bienestar de las instituciones educativas y considerarlas como antecedentes, se destacan las siguientes: el Acuerdo Nacional para disminuir la deserción en la educación superior.Políticas y estrategias para incentivar la permanencia y graduación en educación superior 2013-2014; el acuerdo de la Asociación Colombiana de Universidades (ASCUN) de 2003, sobre políticas nacionales de bienestar universitario; el documento de la Unesco de 2009 sobre asuntos y servicios estudiantiles en la educación superior; la Guía para la implementación del modelo de gestión de permanencia y graduación estudiantil en instituciones de educación superior de 2015, "que establece los lineamientos para implementar un proyecto de permanencia y graduación integral que les ayuda a las Instituciones de Educación Superior (IES) y a los organismos externos, incluidos pares académicos, a evaluar la capacidad institucional para gestionar la permanencia en cumplimiento de los requisitos de acreditación de alta calidad" (p.10); el Decreto 1075 de 2015, que establece las condiciones de calidad de carácter institucional relacionadas con el bienestar universitario, en relación con la información que le suministra el SPADIES.

Así mismo, el Consejo Nacional de Acreditación CNA dentro de sus lineamientos, reglamenta algunos aspectos relacionados con el seguimiento, la evaluación y control de la deserción, permanencia y retención estudiantil y de esta manera comienza la cultura de la autoevaluación en las Instituciones promoviendo programas desde Bienestar universitario. Surge también el documento del Ministerio de Educación, denominado Estrategias para la permananencia en educación superior: Experiencias Significativas en el año 2015, como una apuesta a seguir trabajando más por la prevención de la deserción,entendiendo las múltiples causas que intervienen en este fenómeno y que afectan el proyecto de vida de los estudiantes en particular, y a la calidad de la educación, en general.

Estos documentos coinciden en apuntar a la calidad educativa, para lo que requiere una constante evaluación, regulación y control en el sistema educativo. Sin embargo, la deserción es una problemática que aún siguen enfrentando las universidades por las altas tasas de deserción estudiantil. Este fenómeno no es ajeno al programa académico Licenciatura en Matemáticas, de la Facultad de Ciencias de la Educación de la Universidad del Tolima, del cual nos ocuparemos enseguida no solo para estudiar sus particularidades, sino también, para proponer acciones que favorezcan la permanencia.

\subsection{Características de la deserción del programa de licenciatura en matemáticas}

En este estudio, nos acercamos a la problemática de la deserción caracterizando los actores, conceptos y acciones involucrados, por lo que debemos precisarlos para poder identificarla.

Del glosario del Sistema para la Prevención de la Deserción en las Instituciones de Educación Superior SPADIES, se tuvo en cuenta la siguiente definición:

Deserción: Estado de un estudiante que de manera voluntaria o forzosa no registra matrícula por dos o más períodos académicos consecutivos del programa en el que se matricul ó, y no se encuentra como graduado o retirado por motivos disciplinarios. La deserción es el resultado del efecto de diferentes factores - individuales, académicos, institucionales y socioeconómicos. 
Los conceptos que siguen son tomados de la Guía para la implementación del modelo de gestión de permanencia y graduación estudiantil en instituciones de educación superior(2015):

Caracterización estudiantil: consiste en conocer o determinar las características y el perfil de cada estudiante en aspectos personales, demográficos, académicos, socioeconómicos, familiares, culturales, psicológicos, etc., que permitan entender sus condiciones y factores de vida e identificar sus necesidades reales.

Permanencia estudiantil: comprende la iniciativa permanente de las IES para la generación de estrategias de fortalecimiento en la capacidad institucional, que contribuyan a disminuir las tasas de deserción. De igual manera, se constituye en un elemento importante en la elaboración del plan educativo institucional.

Retención (estudiantil): habilidad de una institución para que el estudiante permanezca en ella desde la admisión hasta la graduación (Berger, Blanco Ramírez y Lyon, 2012) (citado en MEN, 2015, p.196).

Autores clásicos como Tinto (1989), tienen variedad de perspectivas, acerca de la deserción, comprendiendo a los implicados, como por ejemplo el significado de desertar desde el punto de vista individual, la deserción según la perspectiva institucional y la connotación del problema de la deserción desde el punto de vista estatal o nacional. Se busca en este artículo entender estas relaciones y caracterizarlas de manera particular para el programa de licenciatura en matemáticas, aún sabiendo que es un asunto complejo. Este autor, sostiene que "ninguna definición puede captar en su totalidad la complejidad de este fenómeno, quedando en manos de los investigadores la elección de la aproximación que mejor se ajuste a sus objetivos y al problema por investigar" (citado en MEN, 2009, p.20).

Con lo anterior, la Guía para la implementación del modelo de gestión de permanencia y graduación estudiantil en instituciones de educación superior publicada en el año 2015, define la deserción como "el abandono definitivo de las aulas y del programa de formación". Sin embargo, para clasificarlo es necesario establecer por lo menos dos elementos en relación con el tipo de abandono, definiendo este último como "la suspensión de la trayectoria académica del estudiante" (p.15). Las perspectivas de abandono que se consideran, son:

Perspectiva del espacio: se analiza el abandono ocurrido por programa, facultad, institución, región e incluso sistema.

Perspectiva del tiempo: se analiza si la deserción es precoz, temprana o tardía, según el momento del ciclo académico en el cual abandona el estudiante (MEN,2015, p.15).

Para caracterizar la deserción estudiantil del programa Licenciatura en Matemáticas, será necesario describir algunas generalidades del contexto normativo e institucional en relación con las acciones y herramientas para la prevención de la deserción, con las que cuenta la Universidad del Tolima y la Facultad de Ciencias de la Educación, donde se inscribe este programa de modalidad presencial.

Bermúdez (2017) y Sánchez et al (2017), realizan un estudio de la deserción estudiantil en la universidad del Tolima en Colombia, teniendo en cuenta las nueve unidades académicas, que tienen a su vez programas de pregrado en la modalidad presencial, en el que proponen la integración de un modelo que permita hacer seguimiento al problema de la deserción estudiantil en los programas de pregrado. A manera de contextualización, se describen a continuación algunas acciones, en torno a la deserción y a las estrategias de prevención. 
A partir de las políticas públicas, el Ministerio de Educación Nacional en Colombia, se fija metas para disminuir la deserción, por ejemplo: el MEN inicia un trabajo en conjunto con la Universidad de los Andes en el año 2004, donde se seleccionaron unas universidades por su tamaño y complejidad, como muestra piloto para la implementación de la herramienta del Sistema de Prevención y Análisis de la Deserción en las Instituciones de Educación Superior SPADIES, en la cual estaba inmersa la Universidad del Tolima.

Posteriormente, en el año 2010 se realizó una convocatoria para asignar recursos a las universidades del país apoyando el tema de permanencia estudiantil, como estrategia para evitar la deserción. La Universidad del Tolima inicio el proceso de seguimiento a los estudiantes de los niveles 1 y 2, cuyo resultado fue el diagnóstico en su primera fase de estos estudiantes, evidenciando las dificultades en Lectoescritura, Matemáticas, Física y Química; lo que permitió que se iniciara el acompañamiento con monitores, los cuales cursaban entre 8o. y 100. semestre, para la realizaciónn de cursos nivelatorios en estas asignaturas. (citado en Bermúdez, 2017, p.14).

En los procesos de acreditación, los programas académicos a través de los planes de mejoramiento del proceso de autoevaluación, realizan acciones permanentes con el trabajo de los integrantes de los comités curriculares como estrategia, a los estudios de deserción y retención de los estudiantes, realizados mediante el esfuerzo académico de cada programa (Sánchez et al, 2017), este también es el caso de la licenciatura en matemáticas.

Para el caso de la Universidad del Tolima, dentro del Estatuto Estudiantil vigente (Acuerdo No. 006 de 1996, marzo 1 de 1996, pp.10-13) de manera expresa no define desertor, sino la "pérdida de la calidad de estudiante", ( Artículo 9) cuando:

a) No se hace uso del derecho de renovación de matrícula para el período académico siguiente.

b) El estudiante cancela la matrícula de forma voluntaria.

c) Se le cancela la matrícula al estudiante por bajo rendimiento académico.

d) Cuando la cancelación de la matrícula del estudiante obedece a problemas graves de salud.

e) Se le cancela la matrícula al estudiante por sanción disciplinaria.

En todos los casos excepto el inciso c) existe el derecho de reintegro en los siguientes cuatro periodos académicos o semestres, previa aprobación de los consejos de facultad o institutos respectivos. La pérdida de la calidad de estudiante implica la cancelación de todas las asignaturas, lo que equivale a que no han sido cursadas ( p.12).

Cuando a un estudiante al que se le ha cancelado la matrícula por bajo rendimiento académico, reingresa a la universidad, tendrá derecho a la homologación de las asignaturas del anterior programa, en las que haya tenido como mínimo una calificación de 3,5 puntos.

Los derechos de reingreso, una vez se haya perdido la calidad de estudiante, están condicionados a la existencia de los programas y planes de estudio a los que se pretende reingresar y a las modificaciones que puedan haber tenido (p.13).

Esta flexibilidad académica, le permite a los estudiantes que abandonan sus estudios, por alguna causa como por ejemplo, dificultades económicas, o por cumplir con su servicio militar,después de algún tiempo (uno o dos años), regresar al mismo programa.

La vicerrectoría de desarrollo humano, a través de los programas de permanencia estudiantil y de bienestar universitario establece acciones importantes para el acceso, permanencia, motivación y desempeño de los estudiantes, de los que también se ha beneficiado la licenciatura en matemáticas. Respecto al programa de permanencia estudiantil, comprende actividades como: Semana de inducción, Cursos nivelatorios, 
acompañamiento académico I y II semestre, software interactivo y monitores académicos de salvamento, quienes realizarán un acompañamiento constante a los estudiantes de III a VIII semestre, que se encuentren en baja condición académica BRA (Montoya y Ramírez, 2016).

Y en relación con el Programa de Bienestar Universitario, cuenta con la oferta de servicios de: restaurante, residencias estudiantiles y servicios asistenciales, garantizando así, sus derechos de alimentación, vivienda y salud. Así mismo, los estudiantes tienen la posibilidad de acceder a las becas que los exonera de pago de matrícula por rendimiento académico y condiciones socioeconómicas, calamidad, mérito deportivo,méritos artísticos y culturales.

Como puede inferirse de lo anterior, las acciones en la gestión institucional de la Universidad del Tolima, incluyen condiciones que posibilitan el bienestar de los estudiantes, contribuyendo a la formación integral y a los procesos de permanencia estudiantil. Al respecto, el Programa de Permanencia Estudiantil, expresa que:

La Universidad del Tolima a través de convocatoria pública en el año 2010 en asociación con el MEN comenzó a implementar la política de Permanencia Estudiantil denominado "Fortalecimiento de Monitorias Académicas a I y II semestre", cuyo objetivo fundamental es contribuir a la disminución de los índices de deserción estudiantil y repitencia académica de los programas presenciales de la Universidad del Tolima (2016, p.2).

El programa de permanencia estudiantil, se presenta como una de las políticas emprendidas por la Universidad del Tolima por medio de la Vicerrectoría de Desarrollo Humano que contribuye significativamente para generar avances en los retos que impone el fenómeno de la deserción estudiantil (2016,p.2).

Los estudiantes del programa de licenciatura en matemáticas, también tienen la posibilidad de participar en los incentivos del Programa de Jóvenes en Acción del Gobierno Nacional, que busca incentivar y fortalecer la formación para el trabajo de los jóvenes en condición de pobreza y vulnerabilidad, con el fin de activar su ingreso al mercado laboral o incentivar la generación de ingresos autónomos y propender por el mejoramiento de las condiciones de vida de los jóvenes, a través de un modelo de transferencias monetarias condicionadas. Los Convenios Interadministrativos suscritos entre el Departamento para la Prosperidad Social (DPS) y las IES, para la implementación del Programa, prioriza su intervención en los municipios que cuenten con oferta de formación de educación superior en los niveles técnico profesional, tecnólogo y/o profesional universitario en programas de formación con registro calificado vigente y en IES que estén debidamente autorizadas por el MEN (DPS, 2014).

Los estudiantes de la Universidad del Tolima, incluyendo a los estudiantes de la licenciatura en matemáticas, tienen adicionalmente la posibilidad de vincularse con los semilleros de investigación, donde también pueden recibir apoyo económico para asistir a eventos académicos y reciben estímulos académicos, como homologar una o dos asignaturas electivas de su plan de estudios de acuerdo con el número de créditos académicos, recibir formación y acompañamiento para elaborar su proyecto de investigación como opción de grado, además que se constituyen como comunidades de práctica, donde pueden participar estudiantes de todos los semestres y recibir retroalimentación y apoyo entre compañeros antiguos y nuevos y profesores del mismo programa. (Acuerdo $\mathrm{N}^{\circ} 007,2017$ ).

Como se puede notar, la Universidad del Tolima a través de los servicios, proyectos, subsidios y los programas de bienestar y permanencia estudiantil beneficia a los estudiantes del programa de licenciatura en matemáticas; sin embargo, sigue siendo limitados en cobertura, puesto que aunque están las políticas institucionales y las estrategias, no alcanza para todos.

Entender las razones de la deserción o abandono es una situación que tiene diversas causas, en ellas se ponen de manifiesto diversas situaciones de tipo personal, familiar, social, económicas y otras que no pueden ser tan evidentes. Por ello es preciso y adecuado realizar una investigación que permita conocer el por qué los 
estudiantes, que alguna vez estuvieron matriculados en el programa Licenciatura en Matemáticas, ahora son desertores. Además, en la medida en que se disponga de antecedentes, será más fácil la implementación de programas pertinentes y oportunos.

\section{Metodología}

Teniendo en cuenta que la investigación busca caracterizar los factores más influyentes en la deserción de los estudiantes de licenciatura en matemáticas de la Universidad del Tolima, recurrimos a un estudio exploratorio porque permite aumentar el grado de familiaridad con fenómenos relativamente desconocidos, obtener información sobre la posibilidad de llevar a cabo una investigación más completa sobre un contexto particular de la vida real, investigar problemas del comportamiento humano que consideren cruciales los profesionales de determinada área e identificar conceptos o variables promisorias. También es de tipo descriptivo, porque se busca especificar las propiedades importantes de personas, grupos, comunidades o cualquier otro fenómeno que sea sometido a análisis (Dankhe, 1986, citado por Hernández 1994, p.70).

\subsection{Objetivo general}

Determinar las causas por las que los estudiantes del programa Licenciatura en Matemáticas de la Universidad del Tolima, desertan en el periodo de observación de los años 2012-A al 2018-B.

\subsection{Objetivos específicos}

-Identificar los factores y las variables más incidentes en los desertores del programa de licenciatura en matemáticas en el periodo de observación de los años 2012-A al 2018-B.

-Describir algunas variables sociodemográficas y socioeconómicas asociadas al abandono en el programa de Licenciatura en Matemáticas de la Universidad del Tolima.

-Describir y analizar el perfil de los estudiantes desertores de licenciatura en matemáticas en el período académico 2012-A al 2018-B.

\subsection{Diseño de la Investigación}

La investigación se enmarca en una metodología cualitativa, de corte exploratorio y descriptivo, teniendo en cuenta las características del problema y lo que se busca indagar a través de las variables categóricas, que permiten la exploración de significados y sentidos de los factores a través de la descripción, caracterización, interpretación, comprensión de las causas de deserción en el periodo de observación, de los estudiantes del programa licenciatura en matemáticas.

Se realizó un muestreo no probabilístico y se aplicó una encuesta de 15 preguntas, de las cuales seis son cerradas y estaban relacionadas con las dimensiones de Tinto (1989) a través de cuatro variables categóricas: individuales, institucionales, académicas y socieconómicas. Para caracterizar la población, se tendrán en cuenta las respuestas de 56 desertores del programa.

\subsection{Variables de estudio}

La deserción escolar no solo está afectada por las variables académicas, es necesario identificar la relación y la influencia que tienen otras variables externas con este fenómeno, tal como las dimensiones que planteó Tinto(1989), y que se tuvieron en cuenta para realizar las siguientes preguntas en este estudio. 
Tabla 1

Variables categóricas asociadas a la deserción

\begin{tabular}{|c|c|c|c|}
\hline \multicolumn{3}{|c|}{ Variables Sociodemográficas } & Socioeconómicas \\
\hline Individuales & Institucionales & Académicas & \\
\hline $\begin{array}{l}\text { 1. Género } \\
\text { 2. Estado Civil } \\
\text { 3. Con quién } \\
\text { Convivió mientras } \\
\text { estudiaba? } \\
\text { 4. Donde estudia } \\
\text { actualmente } \\
\text { 5. Tipo de estudio. }\end{array}$ & $\begin{array}{l}\text { 1. Regresaría al } \\
\text { programa (sí o no). } \\
\text { 2. Condiciones por las } \\
\text { que Regresaría al } \\
\text { programa: } \\
\text {-Apoyo de matrícula. } \\
\text {-Apoyo académico } \\
\text {-Horarios flexibles } \\
\text {-Apoyo Laboral }\end{array}$ & $\begin{array}{l}\text { 1. Tipo de } \\
\text { bachillerato } \\
\text { 2. Razón por la } \\
\text { que se } \\
\text { matriculó en el } \\
\text { programa. } \\
\text { 3. Regresaría al } \\
\text { programa? }\end{array}$ & $\begin{array}{l}\text { 1. Estrato Social } \\
\text { 2. Sostenimiento } \\
\text { 3. ¿A qué actividad } \\
\text { se dedica } \\
\text { actualmente? }\end{array}$ \\
\hline
\end{tabular}

Fuente: Los autores

Las variables de la tabla 1, a su vez, se pueden agrupar de manera general en dos grupos de variables como se describen a continuación:

Sociodemográficas: Este grupo de variables permite identificar las características sociales de los desertores, en cuanto a sus particularidades individuales, institucionales y académicas.

Socioeconómicas: en este grupo se tendrán en cuenta únicamente las variables que determinan la situación económica de los desertores.

\subsection{Instrumentos y procedimientos}

Se elaboró una encuesta de 15 preguntas, de las cuales seis son cerradas. El instrumento fue validado por 4 expertos, que conforman un grupo de investigación de educación estadística de la Universidad del Tolima. Las preguntas indagan por aspectos sociodemográficos y socioeconómicos de los desertores; las preguntas tienen relación con las variables estudiadas por Tinto (1989).

\subsection{Participantes}

En esta investigación, la población objeto corresponde a los desertores reales (retirados) del programa de licenciatura en matemáticas, quienes han abandonado su proceso de formación en diversos momentos según periodo de observación. En esta ocasión se logró ubicar a 56 desertores reales (Mujeres=22; Hombres=34), quienes aceptaron responder el cuestionario. El procedimiento para ubicar a los desertores fue a través de llamada telefónica y este a su vez nos remitía a otro participante, como el "efecto bola de nieve".

\section{Resultados}

Los resultados que se muestran a continuación, presentan relación entre los objetivos de la investigación y los resultados de las preguntas al cuestionario realizado que, a su vez, permiten verificar que en las respuestas de estas personas hay coherencia con los factores predominantes, de la deserción descritos en el marco teórico. Sin embargo, con algunas peculiaridades que se encontraron para este estudio, como por ejemplo, que cerca del $50 \%$ de los desertores no viven con sus padres, situación que influye en la motivación y dificultades económicas, entre otras razones que se describirán por su relevancia.

Respecto al primer objetivo: Identificar los factores y las variables más incidentes en los desertores del programa de licenciatura en matemáticas en el periodo de observación de los años 2012-A al 2018-B, se encontró lo siguiente: 
Tabla 2

Asociación de variables razón o motivo de deserción del programa y sostenimiento económico durante la época de estudio en la licenciatura en Matemáticas

\begin{tabular}{|l|c|c|c|c|}
\hline \multicolumn{1}{|c|}{$\begin{array}{c}\text { Razones de deserción del programa } \\
\text { Licenciatura en Matemáticas }\end{array}$} & $\begin{array}{c}\text { Ayuda de } \\
\text { otros } \\
\text { Familiares (\%) }\end{array}$ & $\begin{array}{c}\text { Ayuda de } \\
\text { los } \\
\text { Padres (\%) }\end{array}$ & $\begin{array}{c}\text { Recursos de } \\
\text { Trabajo propio } \\
\text { (\%) }\end{array}$ & $\begin{array}{c}\text { Total, motivos } \\
\text { de deserción } \\
\text { (\%) }\end{array}$ \\
\hline Abandona Programa Baja Motivación & 5,36 & 32,14 & 8,93 & 46,43 \\
\hline Abandona Programa Cambio de Carrera & 5,36 & 8,93 & 1,79 & 16,07 \\
\hline $\begin{array}{l}\text { Abandona Programa Problemas Familiares y } \\
\text { Personales }\end{array}$ & 0 & 10,71 & 1,79 & 12,5 \\
\hline Abandona Programa Situación Económica & 1,79 & 16,07 & 7,14 & 25 \\
\hline Total, sostenimiento económico (\%) & 12,5 & 67,86 & 19,64 & 100 \\
\hline
\end{tabular}

Fuente: Los autores

Como se puede apreciar en la tabla 2, la baja motivación es la primera razón para abandonar el programa, le sigue la situación económica y luego el cambio de carrera. Esta baja motivación a su vez, está relacionada con las dificultades que los estudiantes pasan, en cuanto a su sostenimiento económico.

Se observa que la variable sostenimiento de los estudiantes desertores depende en un $67,86 \%$ de sus padres, aunque el $50 \%$ de los estudiantes desertores no viven con ellos y que el el abandono del programa estaría mas bien relacionado con lo académico y lo personal (pago de matrícula, alimentación, vestido, pago de arriendo); mientras que en el $19,6 \%$ de los desertores, el sostenimiento es propio, con las implicaciones que trae, trabajar y estudiar en un programa presencial.

Para dar respuesta al segundo objetivo específico, acerca de "describir algunas variables sociodemográficas y socioeconómicas asociadas al abandono en el programa de Licenciatura en Matemáticas de la Universidad del Tolima", se presentan a continuación los resultados que muestran los mapas perceptuales en las variables asociadas al abandono en el programa de Licenciatura en Matemáticas de la Universidad del Tolima, siendo coherentes con la realidad y las razones detonantes de la deserción presentadas en el marco teórico, lo que además, permite verificar que la respuesta de los desertores fue honesta porque se corresponde con estudios similares realizados en la Universidad del Tolima.

Figura 1

Mapa perceptual de variables sociales y académicas

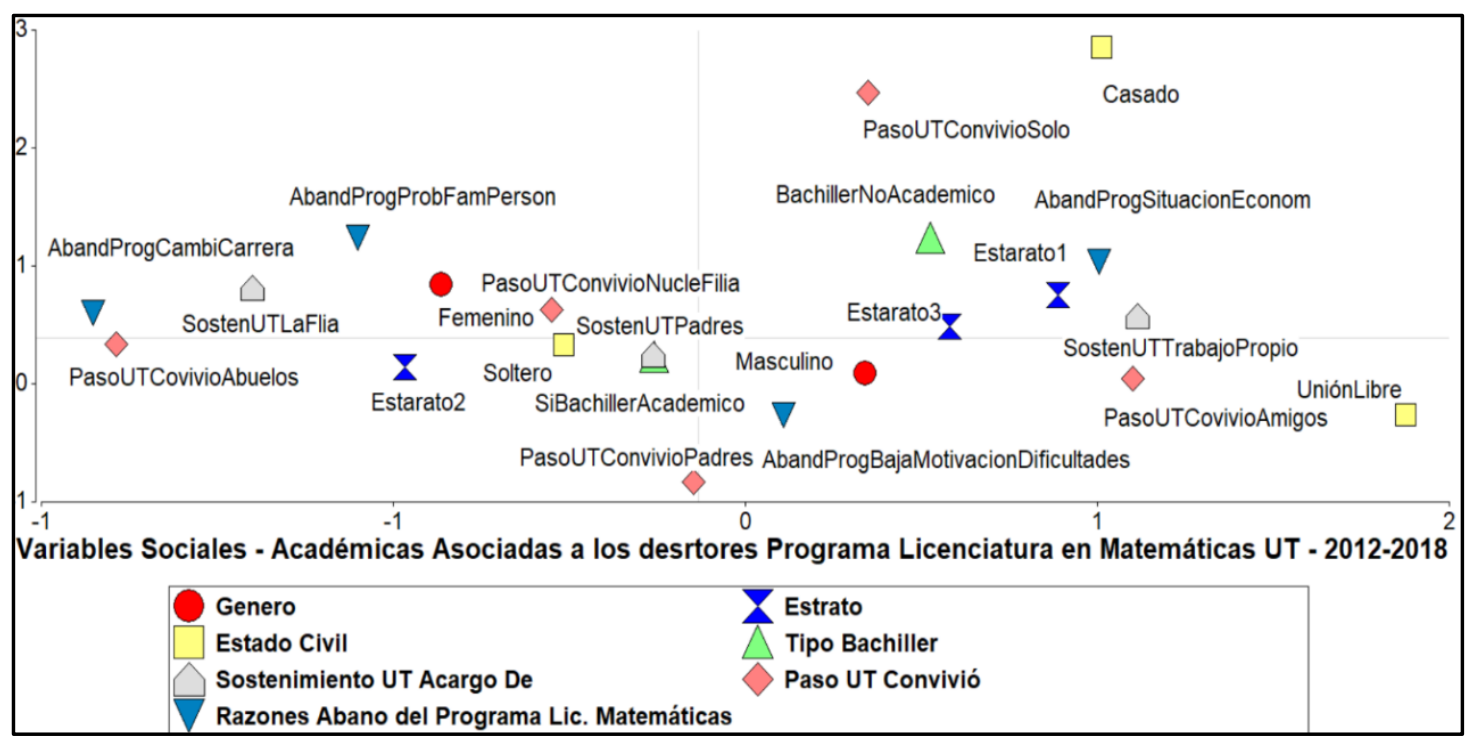

Fuente:Los autores 
La figura 1 permite ver la influencia y relación que han tenido las variables sociodemográficas en la razón de abandono, por parte de los desertores. Se observan las percepciones que se obtuvieron a partir de las opiniones que se recolectaron mediante encuesta, para la caracterización de los desertores con relación a las variables sociales, con características individuales. En cada cuadrante se encontraron variables detonantes asociadas a la razón de abandono:

En el primer cuadrante, se puede ver la razón de abandono por situación económica, como una de las principales asociadas al estrato 1 , desertores que se sostenían con un trabajo y quienes manifestaron que vivían solos mientras estudiaban y en general no son bachilleres académicos.

En el segundo cuadrante del mapa de percepción, se puede apreciar que la razón de abandono de otros desertores fue por problemas familiares y personales y otros, por cambio de carrera, y su sostenimiento estuvo a cargo de la familia y el cuarto cuadrante del mapa perceptual,indica que la principal causa de abandono, según los desertores, es la baja motivación y las dificultades que tuvieron los estudiantes; desertan en mayor proporción, el género masculino y en su mayoría son solteros, teniendo en cuenta que a este programa ingresan estudiantes entre 17 y 18 años de edad.

Figura 2

Mapa perceptual de variables sociales asociadas a la deserción

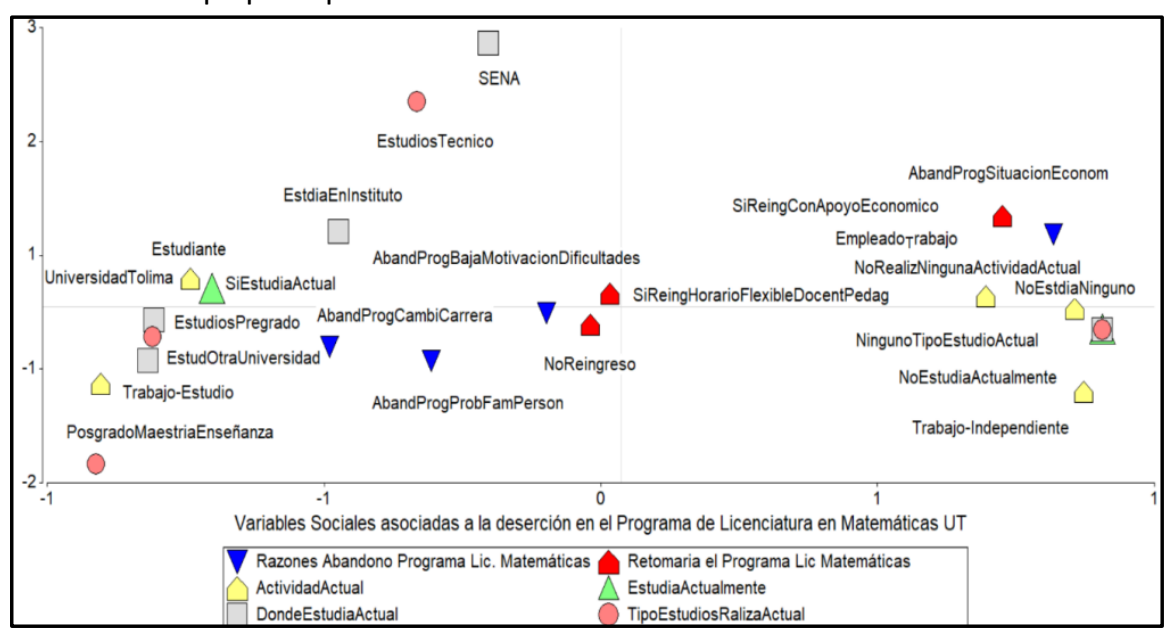

Fuente: Los autores

A los desertores se les preguntó si pensaban regresar al programa de licenciatura en matemáticas y según la figura 2, se evidencia que la mayoría no lo haría porque, o bien se cambió de carrera o por baja motivación y dificultades, relacionadas estas con problemas familiares y personales.

El $16,07 \%$ de los desertores que abandonaron por cambio de carrera, confirmaron que se encontraban estudiando en la universidad del Tolima y el 3.5\% que cambiaron de universidad. Mientras que el $12 \%$ de los desertores indicaron que regresarían si encuentran un empleo o reciben apoyo económico y que no se encuentran realizando alguna actividad actualmente; otra variable asociada, indicó que el $5.4 \%$ de los desertores se encontraban trabajando de manera independiente; informaron tener dificultades por ser un programa presencial, situación que les impide tener un empleo.Estas razones están consideradas como variables individuales que incidieron en la baja motivación.

Y sobre el tercer objetivo específico: Describir y analizar el perfil de los estudiantes desertores de licenciatura en matemáticas en el período académico 2012-A al 2018-B, se encontró que a partir de las categorías de Tinto (1989) y la información suministrada por los mismos desertores, se logra una caracterización muy aproximada 
del perfil de los estudiantes desertores de la licenciatura en matemáticas, de la Universidad del Tolima. Las categorías están asociadas a variables individuales, institucionales, académicas y socioeconómicas.

Respecto a las variables individuales que los afecta se encontró que el $61 \%$ son del género masculino y el 39\% son del género femenino, entre 17 y 18 años de edad, solteros y el $50 \%$ de los desertores no viven con sus padres, porque provienen de otros municipios del Tolima, distintos a la ciudad (Ibagué), donde se encuentra la sede central de la Universidad del Tolima, situación que la Universidad tiene en cuenta, ofreciéndoles residencias universitarias a hombres y mujeres (aunque los cupos son limitados). El 16.07\% manifestó haber desertado para cambiarse de carrera. El $25 \%$ de los desertores, expresó haberlo hecho por falta de apoyo económico y otro $12 \%$ porque los horarios no son flexibles y necesitaban trabajar para ayudar a su sustento. También expresaron dentro de sus razones académicas que el puntaje del ICFES para ingresar a la universidad, había sido muy bajo y que a lo largo de los dos o tres semestres que estuvieron en la universidad, no lograban un rendimiento académico adecuado que les permitiera acceder a una beca. El 75\% de los desertores manifestaron que no regresarían al programa de licenciatura en matemáticas.

La categoría socioeconómica es la segunda característica que más afecta a los desertores con un $25 \%$, porque son estudiantes que provienen de estratos socioeconómicos 1 y 2 y tienen dificultades para el sostenimiento.

\subsection{Análisis y discusión de resultados}

La asociación entre las variables de la figura 1 y las que se observaron en la figura 2 son el resultado del efecto de diferentes factores tanto individuales, como académicas, institucionales y socieconómicas, identificadas en la teoría de Tinto (1989) y son las que aportan información sobre la deserción del programa de licenciatura en matemáticas, aunque tienen un trasfondo particular y es el hecho de que la mayoría de los desertores abandonan por desmotivación y dificultades personales, aspectos que deben ser atendidos al interior del programa de licencitura en matemáticas, por ejemplo, a través de la dirección de programa y o de una consejería profesional.

La encuesta a los desertores puros permitió hacer un análisis a las opiniones en torno a los siguientes factores:

Individuales:disponibilidad de tiempo para estudiar; condiciones personales; cambios en su motivación para continuar con los estudios.

Socioeconómicas: dificultades económicas para cumplir con los costos de matrícula; condiciones laborales y ocupacionales.

Académicas: la razón por la que se matriculó en el programa de licenciatura en matemáticas, no resultó satisfecha en la ptáctica; hubo además dificultad para mantener un promedio que le permitiera participar en las becas por méritos o incetivos de los programas de permanencia estudiantil y bienestar universitario; otras razones: cambio de programa y otras opciones de estudio.

Institucionales: se consideró la importancia de la atención a estudiantes por parte de los profesores y de la dirección de programa. Se requiere más acompañamiento en el desarrollo del plan de estudios, en la matrícula de créditos en cada semestre y el financiamiento estudiantil, para aumentar las oportunidades de continuar en el programa y superar las dificultades.

\section{Conclusiones}

Entre los principales hallazgos están que los factores asociados con el abandono escolar son: las condiciones económicas (falta de beca o apoyo económico), la no insatisfacción de las expectativas que tenían los estudiantes acerca de estudiar la carrera de Licenciatura en Matemáticas y las características individuales influyen en la baja motivación y dificultades, además de ser susceptibles a las políticas y prácticas de los programas de permanencia 
estudiantil y bienestar universitario. A través de estos programas, la Universidad del Tolima busca atender las desventajas académicas y socioeconómicas con las que llegan la mayoría de los estudiantes a una universidad pública, regional y con compromiso social, tal como lo menciona en uno de sus principios: "la universidad actuará con un espíritu solidario a favor de los sectores más vulnerables del conjunto social, y en defensa y desarrollo de la democracia, el interés público, la igualdad, la libertad y la justicia"( Proyecto Educativo Institucional, 2015, p.25). Sin embargo, atender esta problemática no siemre se refleja directamente en lo relacionado con la permanencia estudiantil, aspectos que son confrontados en materia de calidad de la educación. Los recursos asignados a estos programas no siempre son suficientes para satisfacer las necesidades de los estudiantes

Estudios como estos buscan caracterizar el fenómeno de la deserción, entendiendo las particularidades de cada programa, de cada región, de cada universidad, de forma tal, que sirvan como diagnósticos para posibles modelos que se puedan diseñar e implementar por parte de los administrativos y de los profesores, para realizar seguimientos más pertinentes de las variables que afectan los factores socieconómicos y sociodemográficos, con la intención de disminuir la deserción estudiantil.

Las razones de la deserción trascienden el concepto de deserción que presenta el Ministerio de Educación Nacional a través del SPADIES, como el "estado de un estudiante que de manera voluntaria o forzosa no registra matrícula por dos o más períodos académicos consecutivos del programa en el que se matriculó". Se pudo evidenciar que con frecuencia el abandono obedece a razones individuales, por desmotivación y dificultades personales, lo que hace a la deserción un fenómeno que debe ser comprendido a partir de los contextos familiares de los estudiantes y de las posibilidades que le dá el puntaje del examen Saber 11 que realiza el Instituto Colombiano para la Evaluación de la Educación (Icfes) como examen de ingreso a la educación superior, situación desafortunada para el programa de la licenciatura en matemáticas que aunque llega a tener 50 estudiantes matriculados en primer semestre, no se logra la permanencia estudiantil esperada.

\section{Referencias bibliográficas}

Acuerdo $N^{\circ}$ 006. Estatuto Estudiantil. Univeridad del Tolima. 1 de marzo de 1996.

Acuerdo $\mathrm{N}^{\circ} 007$. Por el cual se modifica el reglamento de semilleros de investigación en la Universidad del Tolima. 08 de febrero de 2017.

Bermúdez, N. (2017). Estudio de la deserción estudiantil en las unidades académicas en programas de pregrado de la modalidad presencial de la universidad del Tolima mediante análisis envolvente de datos - DEA y regresión beta. Tesis de maestría. Universidad del Tolima. Colombia.

Departamento para la prosperidad social - DPS (2014). Manual Operativo del Programa Jóvenes en Acción, segunda versión. Dirección del Ingreso Social. Bogotá (Colombia). Recuperado de: https://www.siteal.iiep.unesco.org/sites/default/files/sit_accion_files/co_0415.pdf

DECRETO 1075. Sector Educación. Departamento Administrativo de la Función Pública. Mayo 26 de 2015

Gartner, M. L. \& Gallego, C. (2013).¿Es la permanencia estudiantil un asunto de calidad educativa? Políticas nacionales y gestión institucional para reducir el abandono. Congreso CLABES. Recueprado a partir de http://clabes-alfaguia.org/clabes-2013/comunicaciones_tema3.php

Hernández, S. y otros (1994). Metodología de la investigación. México: Mc Graw Hill, Cap. 4 y 5. Recuperado de https://www.uv.mx/personal/cbustamante/files/2011/06/Metodologia-de-laInvestigaci\%C3\%83\%C2\%B3n_Sampieri.pdf 
MEN.(2009). Deserción estudiantil en la educación superior colombiana. Metodología de seguimiento, diagnóstico y elementos para su prevención. Bogotá: Imprenta Nacional de Colombia.

MEN.(2015). Estrategia para la permanencia en educación superior: Experiencias significativas.Bogotá: Sanmartín Obregón.

MEN.(2015). Guía para la implementación del modelo de gestión de permanencia y graduación estudiantil en instituciones de educación superior. Bogotá: Imprenta nacional de Colombia.

MEN.(2016). Lineamientos de política de bienestar para instituciones de educación superior. Bogotá: QUALIFICAR.

Montoya, G. \& Ramírez, A.M. (2016).Programa de Permanencia Estudiantil. Universidad del Tolima. Vicerrectoría de Desarrollo Humano y Recursos Humanos.

Sistema para la Prevención de la Deserción de la Educación Superior (SPADIES). Ministerio de Educación Nacional. Recuperado de: https://www.mineducacion.gov.co/sistemasinfo/spadies/Zona-deAyuda/254707:Glosario

Sánchez,A; Acuña, J; Rodríguez, M. \& Bermúdez, N.(2017). Estudio de la deserción en programas de pregrado modalidad presencial y a distancia de la Universidad del Tolima. Informe institucional en el marco del convenio $\mathrm{N}^{\circ}$ 80117: Universidad del Tolima. Colombia.

Tinto, Vincent. (1989). Definir la deserción: Una cuestión de perspectiva. Revista de la Educación Superior, 18, (71) <http://publicaciones.anuies.mx/revista/71> [Consulta: marzo de 2019]

Universidad del Tolima.(2015).Proyecto Educativo Institucional (PEI).Segunda edición.Ibagué.

Esta obra está bajo una Licencia Creative Commons Attribución-NoCommercial 4.0 International

(cc) BY-NC 